Article

\title{
Genomic Characterization of Escherichia coli Isolates Belonging to a New Hybrid aEPEC/ExPEC Pathotype O153:H10-A-ST10 eae-beta1 Occurred in Meat, Poultry, Wildlife and Human Diarrheagenic Samples
}

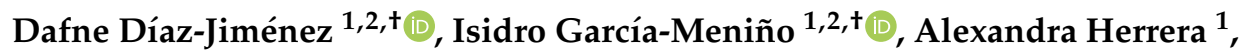 \\ Vanesa García ${ }^{1,2,3}{ }^{1}$, Ana María López-Beceiro ${ }^{4}\left({ }^{1}\right.$, María Pilar Alonso ${ }^{5}$, Jorge Blanco ${ }^{1,2}(\mathbb{D}$ and \\ Azucena Mora 1,2,*iD \\ 1 Laboratorio de Referencia de Escherichia coli (LREC), Departamento de Microbioloxía e Parasitoloxía, \\ Facultade de Veterinaria, Universidade de Santiago de Compostela (USC), 27002 Lugo, Spain; \\ dafne.diaz@usc.es (D.D.-J.); isidro.garcia@usc.es (I.G.-M.); alex.herrera.est@gmail.com (A.H.); \\ vanesag.menendez@usc.es (V.G.); jorge.blanco@usc.es (J.B.) \\ 2 Instituto de Investigación Sanitaria de Santiago de Compostela (IDIS), 15706 Santiago, Spain \\ 3 Department of Veterinary and Animal Sciences, Faculty of Health and Medical Sciences, University of \\ Copenhagen, 1870 Frederiksberg, Denmark \\ 4 Departamento de Anatomía, Produción Animal e Ciencias Clínicas Veterinarias, Facultade de Veterinaria, \\ Universidade de Santiago de Compostela (USC), 27002 Lugo, Spain; anam.lopez.beceiro@usc.es \\ 5 Unidade de Microbioloxía, Hospital Universitario Lucus Augusti (HULA), 27003 Lugo, Spain; \\ Pilar.Alonso.Garcia@sergas.es \\ * Correspondence: azucena.mora@usc.es; Tel.: +34-982822110 \\ + These authors contributed equally to this work.
}

Received: 30 March 2020; Accepted: 15 April 2020; Published: 17 April 2020

\begin{abstract}
Different surveillance studies (2005-2015) in northwest Spain revealed the presence of eae-positive isolates of Escherichia coli $\mathrm{O} 153: \mathrm{H} 10$ in meat for human consumption, poultry farm, wildlife and human diarrheagenic samples. The aim of this study was to explore the genetic and genomic relatedness between human and animal/meat isolates, as well as the mechanism of its persistence. We also wanted to know whether it was a geographically restricted lineage, or whether it was also reported elsewhere. Conventional typing showed that 32 isolates were O153:H10-A-ST10 fimH54, fimAv $v_{M T 78}$, traT and eae-beta1. Amongst these, 21 were CTX-M-32 or SHV-12 producers. The PFGE $X b a I$-macrorestriction comparison showed high similarity $(>85 \%)$. The plasmidome analysis revealed a stable combination of IncF (F2:A-:B-), IncI1 (STunknown) and IncX1 plasmid types, together with non-conjugative Col-like plasmids. The core genome investigation based on the cgMLST scheme from EnteroBase proved close relatedness between isolates of human and animal origin. Our results demonstrate that a hybrid MDR aEPEC/ExPEC of the clonal group O153:H10-A-ST10 (CH11-54) is circulating in our region within different hosts, including wildlife. It seems implicated in human diarrhea via meat transmission, and in the spreading of ESBL genes (mainly of CTX-M-32 type). We found genomic evidence of a related hybrid aEPEC/ExPEC in at least one other country.
\end{abstract}

Keywords: Escherichia coli; ESBL; hybrid pathotype; ExPEC; EPEC; MDR; ST10; O153; EnteroBase

\section{Introduction}

Escherichia coli is a normal inhabitant of the human and animal intestinal tract. However, E. coli can also act as a pathogen in a broad range of conditions, from enteric diseases to extraintestinal infections, such as urinary tract infection (UTI) and sepsis, among others. Strains that cause enteric disease or 
diarrhea are called diarrheagenic E. coli (DEC), which includes six major categories characteristically defined by certain pathotype-specific virulence markers [1,2]. Thus, the enteropathogenic E. coli (EPEC) category is typically carrier of the eae gene, as part of the pathogenicity island locus of enterocyte effacement (LEE), codifying a protein called intimin. The intimin is responsible for the intimate adherence of the bacteria to the enterocyte membranes and, eventually, for the attaching and effacing (AE) lesion of the brush-border microvilli [3]. The variable C-terminal-encoding sequence of eae defines more than 30 distinct intimin types and subtypes associated with tissue tropism [4,5]. EPEC are further classified as typical (tEPEC) when they carry an EPEC adherence factor (EAF) plasmid that encodes adherence mediated by the bundle forming pilus (BFP), while atypical EPEC (aEPEC) produce the AE lesion, but do not express BFP [4,6]. Currently, aEPEC isolates are emerging enteropathogens detected worldwide and isolated from different niches (animal species, environment, and food samples), while the main reservoir of tEPEC isolates are humans $[7,8]$. On the other hand, E. coli that cause extraintestinal infections are referred to as extraintestinal pathogenic E. coli (ExPEC), which includes a heterogeneous group of pathotypes defined by isolation from infections outside the intestinal tract: uropathogenic E. coli (UPEC), avian pathogenic E. coli (APEC), and neonatal meningitis E. coli (NMEC). A variety of specific virulence genes have been described in ExPEC (adhesins, protectins, siderophores, toxins, hemolysins, polysaccharide antigens, invasins, colicins, etc.) [1,2]. However, even though certain virulence traits and phylogenetic groups have been proposed to characterize ExPEC $[9,10]$, no set of genes can be used to unequivocally distinguish them from commensal E. coli. In fact, ExPEC strains can commensally colonize the human intestine, which in turn, can serve as reservoir [11]. Antimicrobial resistance is a serious global concern which involves the health care system, food production and environmental integrity [12]. In fact, it is assumed that antimicrobial drugs used in the livestock sector play an important role in the spread of extended-spectrum beta-lactamases (ESBL)-producing E. coli throughout the food chain to humans $[13,14]$. The genomic plasticity of $E$. coli is the consequence of the important role played by mobile genetic elements (MGEs) such as plasmids, bacteriophages, pathogenicity islands, transposons and insertion sequence elements in the evolution of the bacteria [15]. As a result, hybrid E. coli pathotypes unpredictably emerge, given the mobility of most of the genes encoding virulence and antimicrobial resistance (AMR) $[15,16]$. Since 2011, when a novel Shiga-toxin-producing E. coli (STEC) belonging to serotype O104:H4, with virulence features (VF) common to the enteroaggregative E. coli (EAggEC) and CTX-M-15 producer was identified as the one involved in the large German outbreak [17], the concept of pathotype has been questioned. Currently, classical and new approaches, such as whole genome sequencing (WGS), are being used to enhance the understanding of the evolution of this highly adaptable species $[16,18]$.

Different in-house surveillance studies in northwest Spain (2005-2015) revealed the presence of eae-positive isolates of E. coli O153:H10, many of them ESBL-producers, in meat for human consumption, wildlife, and avian farm environments. We also found them involved in human diarrhea. The aim of this study was to explore the genetic and genomic characterization relatedness between human and animal/meat isolates, as well as to gain knowledge in the mechanism that might be playing a role in its persistence. We also wanted to know whether it was a geographically restricted E. coli lineage, or whether it was also reported somewhere else.

\section{Results}

Thirty-two eae-positive E. coli (21 ESBL and 11 non-ESBL) belonging to the serotype O153:H10 constituted the collection of the study: 14 from human stools, 8 from beef meat, 7 from chicken meat, and 1 each from pork meat, wildlife (fox feces) and a poultry farm environment sample, as shown in Table S1. They were detected within different surveys from 2005 to 2015 based on the following findings. First, we noticed the recovery of eae-positive isolates with the same intimin type. Second, many of them were ESBL-producers (coming from surveillance studies on ESBL-producing E. coli in animal sources). Third, they belonged to the O153 serogroup. Fourth, we found them within the human collection in the retrospective analysis. Fifth, the isolates also carried ExPEC virulence genes. 


\subsection{Conventional Typing}

Table 1 summarizes the main traits obtained by conventional typing for the 32 isolates. All were positive for the intimin eae-beta1, but negative for $b f p A$ gene, conforming the aEPEC pathotype. Other virulence genes defining verotoxigenic (VTEC), enteroinvasive (EIEC), enteroaggregative (EAggEC) or enterotoxigenic (ETEC) pathotypes were not detected. Interestingly, the investigation of virulence traits associated with ExPEC showed that the fim $A v_{M T 78}$ gene, which is a virulence locus that codify a fim $A$ variant MT78 of type 1 fimbriae [19], was present in all isolates. In addition, the traT gene that codifies an outer membrane protein implicated in serum survival [20] was also determined in 17 of the isolates, as shown in Table 1. Finally, all the isolates were assigned to the clonal group O153:H10-A-ST10 (CH11-54) by means of the serotyping, phylogroup, MLST and clonotyping.

The highest rates of AMR were to: ampicillin $(75 \% ; 24 / 32)$, cefuroxime $(68.7 \% ; 22 / 32)$, cefotaxime $(65.6 \%, 21 / 32)$, ceftazidime $(65.6 \%, 21 / 32)$, cefepime $(59.4 \%, 19 / 32)$ and gentamicin $(59.4 \%, 19 / 32)$. The ESBL-typing of the 21 positive isolates determined that 19 were CTX-M-32 and two SHV-12 producers, as shown in Table 1.

The comparison of the XbaI-macrorrestriction profiles obtained by pulsed field gel electrophoresis (PFGE) of the ESBL-producing aEPEC isolates revealed high similarity. Thus, all but one clustered with an identity $>85 \%$ in the dendrogram shown in Figure 1 . It is of note that three human clinical isolates, recovered in different years, clustered each with a fox ( $95.2 \%$ of similarity) and with two beef meat isolates $(100 \%$ and $97.6 \%$ of similarity, respectively).

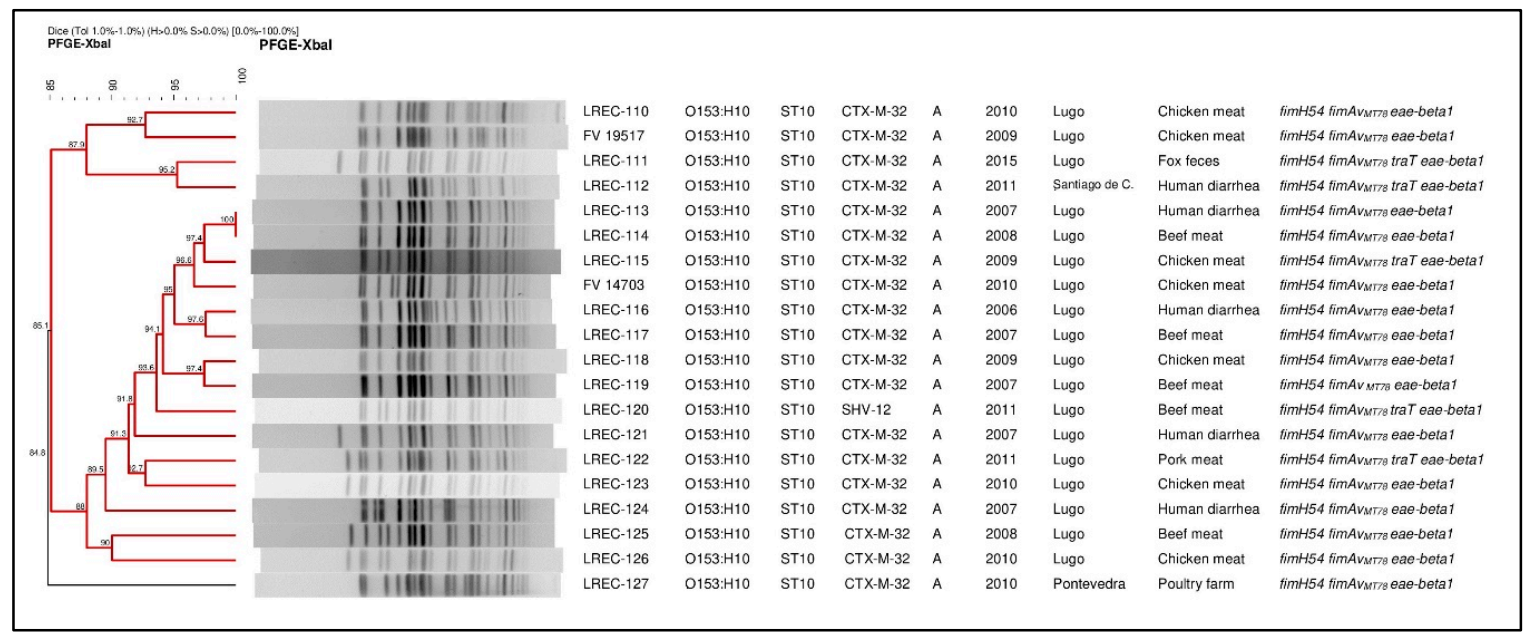

Figure 1. Pulsed field gel electrophoresis (PFGE) of XbaI-digested DNA from 20 ESBL-producing aEPEC isolates of the clonal group O153:H10-A-ST10 (one autodigested). On the right of the dendrogram: Isolate designation, O:H serotype, ST, ESBL type, phylogroup, year of isolation, geographic origin, source and virulence-gene profile. 
Table 1. Phenotypic and genotypic characterization of 32 aEPEC O153:H10-A-ST10 (CH11-54) isolates.

\begin{tabular}{|c|c|c|c|c|c|c|}
\hline Sample Origin & Code $^{a}$ & Year & Geographic Origin & Virulence Gene Profile & Resistance Profile $^{\mathrm{b}}$ & $b l a_{\mathrm{ESBL}}$ Type \\
\hline Pork meat & ${ }^{*}$ LREC-122 & 2011 & Lugo & fimH54, fimAv $v_{M T 78}$, traT, eae-beta 1 & AMP, CXM, CTX, CAZ, FEP, GEN & CTX-M-32 \\
\hline Chicken meat & *LREC-115 & 2009 & Lugo & fimH54, fimAv $v_{M T 78}$, traT, eae-beta1 & AMP, CXM, CTX, CAZ, FEP, GEN, TOB & CTX-M-32 \\
\hline Chicken meat & FV 19517 & 2009 & Lugo & fimH54, fimAv ${ }_{M T 7}$, eae-beta1 & AMP, CXM, CTX, CAZ, FEP, GEN & CTX-M-32 \\
\hline Chicken meat & ${ }^{*}$ LREC-118 & 2009 & Lugo & fimH54, fimAv $v_{M T 78}$, eae-beta1 & AMP, CXM, CTX, CAZ, FEP, GEN & CTX-M-32 \\
\hline Chicken meat & ${ }^{*}$ LREC-110 & 2010 & Lugo & fimH54, fimAv $v_{M T 78}$, eae-beta1 & AMP, CXM, CTX, CAZ, FEP, GEN & CTX-M-32 \\
\hline Chicken meat & FV 14703 & 2010 & Lugo & fimH54, fimAv $v_{M T 78}$, eae-beta1 & AMP, CXM, CTX, CAZ, FEP, GEN, TOB, FOF & CTX-M-32 \\
\hline Chicken meat & LREC-126 & 2010 & Lugo & fimH54, fimAv $v_{M T 78}$, eae-beta1 & AMP, CXM, CTX, CAZ, FEP, GEN, TOB & СТХ-M-32 \\
\hline Chicken meat & *LREC-123 & 2010 & Lugo & fimH54, fimAv ${ }_{M T 78}$, eae-beta1 & AMP, CXM, CTX, CAZ, FEP, GEN, TOB & CTX-M-32 \\
\hline Beef meat & ${ }^{*}$ LREC-119 & 2007 & Lugo & fimH54, fimAv MT78, eae-beta1 & AMP, CXM, CTX, CAZ, FEP, GEN & CTX-M-32 \\
\hline Beef meat & ${ }^{*}$ LREC-117 & 2007 & Lugo & fimH54, fimAv $v_{M T 78}$, eae-beta1 & AMP, CXM, CTX, CAZ, FEP, GEN & CTX-M-32 \\
\hline Beef meat & $4-3 a$ & 2007 & Lugo & fimH54, fimAv ${ }_{M T 78}$, traT, eae-beta 1 & AMP, CXM, CTX, CAZ & SHV-12 \\
\hline Beef meat & $85-5 a$ & 2008 & Lugo & fimH54, fimAvivi ${ }_{M T 7}$, traT, eae-beta1 & AMP, GEN & - \\
\hline Beef meat & ${ }^{*}$ LREC-125 & 2008 & Lugo & fimH54, fimAv $v_{M T 78}$, eae-beta1 & AMP, CXM, CTX, FEP & CTX-M-32 \\
\hline Beef meat & ${ }^{*}$ LREC-114 & 2008 & Lugo & fimH54, fimAv ${ }_{M T 78}$, eae-beta1 & AMP, CXM, CTX, CAZ, FEP, GEN, TOB & CTX-M-32 \\
\hline Beef meat & $65-6 a$ & 2009 & Lugo & fimH54, fimAv $v_{M T 78}$, traT, eae-beta 1 & - & - \\
\hline Beef meat & ${ }^{*}$ LREC-120 & 2011 & Lugo & fimH54, fim Av ${ }_{M T 78}$, traT, eae-beta 1 & AMP, CXM, CTX, CAZ, FEP & SHV-12 \\
\hline Wildlife (Fox) & ${ }^{*}$ LREC-111 & 2015 & Lugo & fimH54, fim Av ${ }_{M T 78}$, traT, eae-beta 1 & AMP, CXM, CTX, CAZ, FEP, GEN, TOB & CTX-M-32 \\
\hline Poultry farm & ${ }^{*}$ LREC-127 & 2010 & Pontevedra & fimH54, fimAvivis8, eae-beta1 & AMP, CXM, CTX, CAZ, FEP, GEN & CTX-M-32 \\
\hline Human & ${ }^{*}$ LREC-116 & 2006 & Lugo & fimH54, fimAv ${ }_{M T 78}$, eae-beta1 & AMP, CXM, CTX, CAZ, FEP, GEN, TOB & CTX-M-32 \\
\hline Human & *LREC-113 & 2007 & Lugo & fimH54, fimAv $v_{M T 78}$, eae-beta1 & AMP, CXM, CTX, CAZ, FEP, GEN, TOB & CTX-M-32 \\
\hline Human & ${ }^{*}$ LREC-121 & 2007 & Lugo & fimH54, fimAv ${ }_{M T 78}$, eae-beta1 & AMP, CXM, CTX, CAZ, FEP, GEN, TOB & CTX-M-32 \\
\hline Human & ${ }^{*}$ LREC-124 & 2007 & Lugo & fimH54, fimAv ${ }_{M T 78}$, eae-beta1 & AMP, CXM, CTX, CAZ, FEP, GEN, TOB & CTX-M-32 \\
\hline Human & 31952. 07 & 2007 & Lugo & fimH54, fimAv ${ }_{M T 78}$, traT, eae-beta 1 & - & - \\
\hline Human & 32651.07 & 2007 & Lugo & fimH54, fimAv $v_{M T 78}$, traT, eae-beta 1 & NAL, CIP & - \\
\hline Human & 32884. 07 & 2007 & Lugo & fimH54, fimAvivi ${ }_{M T 8}$, traT, eae-beta1 & AMP, CXM, CAZ, AMC, SXT & - \\
\hline Human & 34535.07 & 2007 & Lugo & fimH54, fimAvivT78, traT, eae-beta1 & NAL, CIP & - \\
\hline Human & 39044.07 & 2007 & Lugo & fimH54, fimAv $v_{M T 78}$, traT, eae-beta 1 & - & - \\
\hline Human & 21011. 08 & 2008 & Lugo & fimH54, fimAvivT78, traT, eae-beta1 & - & - \\
\hline Human & 38506. 08 & 2008 & Lugo & fimH54, fimAv $v_{M T 78}$, traT, eae-beta 1 & CIP & - \\
\hline Human & 40237. 08 & 2008 & Lugo & fimH54, fimAv ${ }_{M T 78}$, traT, eae-beta1 & NAL, CIP & - \\
\hline Human & ${ }^{*}$ LREC-112 & 2011 & Santiago de Compostela & fimH54, fimAv ${ }_{M T 78}$, traT, eae-beta1 & AMP, CXM, CTX, CAZ, FEP, NAL & CTX-M-32 \\
\hline Human & 55515.12 & 2012 & Lugo & fimH54, fimAv ${ }_{M T 78}$, traT, eae-beta 1 & AMP, GEN & - \\
\hline
\end{tabular}

a Strains marked with $\left(^{*}\right)$ were further analyzed by WGS; ${ }^{\text {b }}$ ampicillin (AMP), amoxicillin/clavulanic acid (AMC), cefuroxime (CXM), ceftazidime (CAZ), cefotaxime (CTX), cefepime (FEP), cefoxitin (FOX), gentamicin (GEN), tobramycin (TOB), fosfomycin (FOF), sulfamethoxazole/trimethoprim (SXT), ciprofloxacin (CIP) and nalidixic acid (NAL). 


\subsection{Whole Genome Sequencing (WGS)}

Based on the high similarity shown by PFGE and to further investigate the virulence profile, resistome, plasmid content and relatedness, 17 representative aEPEC/ExPEC isolates were WG sequenced. The de novo assembled contigs were typed in silico using the EnteroBase tools, as shown in Table S2, as well as the Center for Genomic Epidemiology (CGE) databases, also shown in Table 2.

SerotypeFinder and EnteroBase predictions corroborated $\mathrm{O}$ and $\mathrm{H}$ antigens, with the exception of LREC-120 and LREC-121, for which O153 was solved by serotyping. MLST (CGE and EnteroBase), CHTyper and ClermonTyping also confirmed conventional data for ST (10), CH (11-54) and phylogroup (A), shown in Table 2 and Table S2. Additionally, the wgST, cgST, and rST of the genomes were determined using the schemes of EnteroBase, shown in Table S2. Whole genome multilocus sequence typing (WgMLST) and core genome multilocus sequence typing (cgMLST) are powerful schemes with extreme and high resolution, respectively. cgMLST is defined as MLST based on the core genome, whereas wgMLST is based on a non redundant set of genes across a species, similar to a 'pan-genome'. In EnteroBase, the number of core loci included in the cgMLST scheme for E. coli is 2,512 and 25,002 in the wgMLST. Different cgSTs and wgSTs were assigned to each of the 17 genomes analyzed, while rST (medium resolution; 53 loci) was the same (2021) for all genomes, excluding LREC-127 (58738), as shown in Table S2.

VirulenceFinder corroborated the hybrid pathotype nature of the isolates, predicting in all genomes the eae gene (intimin) together with other components encoded in the LEE pathogenity island, as well as the increased serum survival gene iss recognized for its role in ExPEC virulence [21]. The ast $A$ gene, which encodes the heat-stable enterotoxin 1, was also present in all 17 isolates (Table 2).

ResFinder identified the genes associated to resistances observed in vitro (acquired resistances for beta-lactams, aminoglycosides, and point mutations for quinolones), only, the bla $a_{C T X-M-32}$ was not predicted in silico for LREC-112 and LREC-119, but by conventional sequencing. Furthermore, ResFinder determined other acquired resistances which had not been tested in vitro, such as to phenicols and macrolides in all genomes, and to tetracyclines in 16 out of the 17 genomes, as shown in Table 2.

Based on the replicon identification, PlasmidFinder revealed a homogenous profile of four or five plasmid types. Thus, the concomitant presence of IncF (F2:A-:B-), IncI1 (STunknown) and IncX1, together with non-conjugative Col156-like plasmids, was detected in 15 of 17 genomes. Four of those 15 genomes were also carriers of Col (MG828)-like plasmids, as shown in Table 2.

In the asymmetric distance matrix on the cgMLST scheme from EnteroBase, based on the presence or absence of 2,513 genes, the 17 genomes showed $<20$ differences (range 5-19) in relation to the human diarrheagenic isolate LREC-113, as displayed in Table 3 and Figure 2. We also looked into the static Hierarchical Clustering (HierCC) designations in EnteroBase. The 17 genomes were assigned into the same HierCC HC50 (37600), which means all strains in this cluster have links no more than 50 alleles apart. Furthermore, using HC20, three human genomes (LREC-113, LREC-116, LREC-124) and two beef meat (LREC-119, LREC-125) clustered together (37606) with links no more than 20 alleles apart, as shown in Table S3. A dendrogram was also built in EnteroBase based on the SNPs of the core genomic regions present in 90\% of the compared genomes, and using LREC-113 as reference, downloaded, and modified with FigTree v1.4.3 as shown in Figure 3. Within 1,068 variant sites, the number of SNPs was $<62$ for 13 of the 17 genomes, as displayed in Table S3. 
Table 2. In silico characterization of 17 Escherichia coli genomes from the study collection using Center for Genomic Epidemiology (CGE) databases and ClermonTyping (in red, results obtained only by conventional typing).

\begin{tabular}{|c|c|c|c|c|c|c|c|}
\hline Code & Serotype $^{1}$ & Phylo Group ${ }^{2}$ & $\underset{3}{\text { CHType }}$ & $\mathrm{ST}^{4}$ & $\begin{array}{l}\text { Plasmid Content Inc } \\
\text { Group (pMLST) }{ }^{5}\end{array}$ & $\begin{array}{l}\text { Acquired Resistances (black) and } \\
\text { Point Mutations (blue) }{ }^{6}\end{array}$ & Virulence Genes 7,8 \\
\hline LREC-110 & O153:H10 & A & $11-54$ & 10 & $\begin{array}{c}\text { IncF (F2:A-:B-) } \\
\text { IncI1 (STunknown) } \\
\text { IncX1 } \\
\text { Col156 }\end{array}$ & $\begin{array}{l}\text { bla }_{C T X-M-32}, b l a_{T E M-1 A} ; \operatorname{aac}(3)-I I a \\
\quad \text { aadA1; } \operatorname{cat} A 1 ; \operatorname{mdf}(A) ; \operatorname{tet}(A)\end{array}$ & $\begin{array}{l}\text { ast } A, \text { eae, } \operatorname{esp} A, \operatorname{esp} B, \operatorname{esp} F \\
\text { gad, iss, } m c h F, \text { nle A, tccP, tir }\end{array}$ \\
\hline LREC-111 & O153:H10 & A & $11-54$ & 10 & $\begin{array}{c}\text { IncF (F2:A-:B-) } \\
\text { IncI1 (STunknown) } \\
\text { IncX1 } \\
\text { Col156 } \\
\text { Col (MG828) }\end{array}$ & $\begin{array}{l}\text { bla }_{C T X-M-32}, b l a_{T E M-1 A} ; a a c(3)-I I a \\
\quad \text { aadA1; } \operatorname{cat} A 1 ; \operatorname{mdf}(A) ; \operatorname{tet}(A)\end{array}$ & $\begin{array}{c}\text { ast } A, \text { eae, esp } A, \operatorname{esp} B, \text { gad, iss, } \\
\text { mchF, nle A, tccP, tir }\end{array}$ \\
\hline LREC-112 & O153:H10 & A & $11-54$ & 10 & $\begin{array}{c}\text { IncF (F2:A-:B-) } \\
\text { IncX1 } \\
\text { Col156 } \\
\text { Col (MG828) }\end{array}$ & $\frac{\text { bla }_{C T X-M-32 ;} \text { aadA1; catA1; } \operatorname{mdf}(A) ;}{\operatorname{tet}(A) ; \operatorname{gyr} A \mathrm{~S} 83 \mathrm{~L}}$ & $\begin{array}{l}\text { ast } A, \text { eae, } \operatorname{esp} A, \operatorname{esp} B, \operatorname{esp} F \\
\text { gad, iss, } m c h F, \text { nle } A, t c c P\end{array}$ \\
\hline LREC-113 & O153:H10 & A & $11-54$ & 10 & $\begin{array}{c}\text { IncF (F2:A-:B-) } \\
\text { IncI1 (STunknown) } \\
\text { IncX1 } \\
\text { Col156 }\end{array}$ & $\begin{array}{l}\text { bla }_{C T X-M-32}, b l a_{T E M-1 A} ; \operatorname{aac}(3)-I I a \\
\quad \operatorname{aad} A 1 ; \operatorname{cat} A 1 ; \operatorname{mdf}(A) ; \operatorname{tet}(A)\end{array}$ & $\begin{array}{c}\text { ast } A, \text { eae, esp } A, \operatorname{esp} B, \operatorname{esp} F \\
\text { gad, iss, mchF, tir }\end{array}$ \\
\hline LREC-114 & O153:H10 & $\mathrm{A}$ & $11-54$ & 10 & $\begin{array}{c}\text { IncF (F2:A-:B-) } \\
\text { IncI1 (STunknown) } \\
\text { IncX1 } \\
\text { Col156 } \\
\text { Col (MG828) }\end{array}$ & $\begin{array}{l}\text { bla }_{C T X-M-32,} \text { bla } \\
\quad \text { aad } 1 \text { TEM-1A; } ; \operatorname{cat} A 1 ; \operatorname{mdf}(A) ; \operatorname{tet}(A)\end{array}$ & $\begin{array}{c}\text { ast } A, \text { eae, } \operatorname{esp} A, \operatorname{espB}, \operatorname{espF} \\
\text { gad, iss, mchF, nleA, tir }\end{array}$ \\
\hline LREC-115 & O153:H10 & A & $11-54$ & 10 & $\begin{array}{c}\text { IncF (F2:A-:B-) } \\
\text { IncI1 (STunknown) } \\
\text { IncX1 } \\
\text { Col156 }\end{array}$ & $\begin{array}{l}\text { bla }_{C T X-M-32}, b l a_{T E M-1 A} ; \operatorname{aac}(3)-I I a \\
\quad \text { aadA1; } \operatorname{cat} A 1 ; \operatorname{mdf}(A) ; \operatorname{tet}(A)\end{array}$ & $\begin{array}{l}\text { ast } A, \text { eae, espA, espB, espF, } \\
\text { gad, iss, mchF, nleA, tccP, tir }\end{array}$ \\
\hline LREC-116 & O153:H10 & A & $11-54$ & 10 & $\begin{array}{c}\text { IncF (F2:A-:B-) } \\
\text { IncI1 (STunknown) } \\
\text { IncX1 } \\
\text { Col156 }\end{array}$ & $\begin{array}{l}\text { bla }_{C T X-M-32}, b l a_{T E M-1 A} ; \operatorname{aac}(3)-I I a \\
\quad \text { aadA1; } \operatorname{cat} A 1 ; \operatorname{mdf}(A) ; \operatorname{tet}(A)\end{array}$ & $\begin{array}{c}\text { ast } A, \text { eae, esp } A, \operatorname{esp} B, \text { gad, iss, } \\
\text { mchF, tccP, tir }\end{array}$ \\
\hline
\end{tabular}


Table 2. Cont

\begin{tabular}{|c|c|c|c|c|c|c|c|}
\hline Code & Serotype $^{1}$ & Phylo Group ${ }^{2}$ & $\underset{3}{\text { CHType }}$ & $\mathrm{ST}^{4}$ & $\begin{array}{l}\text { Plasmid Content Inc } \\
\text { Group (pMLST) }{ }^{5}\end{array}$ & $\begin{array}{l}\text { Acquired Resistances (black) and } \\
\text { Point Mutations (blue) }{ }^{6}\end{array}$ & Virulence Genes 7,8 \\
\hline LREC-117 & O153:H10 & A & $11-54$ & 10 & $\begin{array}{c}\text { IncF (F2:A-:B-) } \\
\text { IncI1 (STunknown) } \\
\text { IncX1 } \\
\text { Col156 }\end{array}$ & $\operatorname{bla}_{C T X-M-32} ; \operatorname{aad} A 1 ; \operatorname{mdf}(A) ; \operatorname{tet}(A)$ & $\begin{array}{c}\text { ast } A, \text { eae, esp } A, \operatorname{esp} B, \text { gad, iss, } \\
\text { mchF, tccP, tir }\end{array}$ \\
\hline LREC-118 & O153:H10 & A & $11-54$ & 10 & $\begin{array}{c}\text { IncF (F2:A-:B-) } \\
\text { IncI1 (STunknown) } \\
\text { IncX1 } \\
\text { Col156 } \\
\text { Col(MG828) }\end{array}$ & $\begin{array}{l}\text { bla }_{C T X-M-32,} \operatorname{bla}_{T E M-1 A} ; \operatorname{aac}(3)-I I a, \\
\quad \text { aadA1; } \operatorname{cat} A 1 ; \operatorname{mdf}(A) ; \operatorname{tet}(A)\end{array}$ & $\begin{array}{l}\text { ast } A, \text { eae, esp } A, \operatorname{esp} B, \operatorname{esp} F \\
\text { gad, iss, } m c h F, \text { nle } A, t c c P, \text { tir }\end{array}$ \\
\hline LREC-119 & O153:H10 & A & $11-54$ & 10 & Col156 & $\underline{b l a_{C T X-M-32}}, \operatorname{aadA1} ; \operatorname{cat} A 1 ; \operatorname{mdf}(A)$ & $\begin{array}{c}\text { ast } A, \text { eae, esp } A, \operatorname{esp} B, \text { gad, iss, } \\
\text { nle A, tccP, tir }\end{array}$ \\
\hline LREC-120 & $\underline{\mathrm{O} 153}: \mathrm{H} 10$ & A & $11-54$ & 10 & $\begin{array}{c}\text { IncI1 (ST22-CC2) } \\
\text { IncQ1 } \\
\text { IncX1 } \\
\text { Col156 } \\
\text { Col (MG828) }\end{array}$ & $\begin{array}{c}\text { bla }_{S H V-12} ; \operatorname{aad} A 1, \text { aadA2; } \operatorname{cat} A 1, \operatorname{cml} A 1 \\
m d f(A) ; \operatorname{sul} 3 ; \operatorname{tet}(A)\end{array}$ & $\begin{array}{c}\text { ast } A, \text { eae, esp } A, \operatorname{esp} B, \text { gad, iss, } \\
\text { mchF, nle A, tccP, tir }\end{array}$ \\
\hline LREC-121 & $\underline{\mathrm{O} 153}: \mathrm{H} 10$ & A & $11-54$ & 10 & $\begin{array}{c}\text { IncF (F2:A-:B-) } \\
\text { IncI1 (STunknown) } \\
\text { IncX1 } \\
\text { Col156 }\end{array}$ & $\begin{array}{l}\text { bla }_{C T X-M-32}, b l a_{T E M-1 A} ; a a c(3)-I I a, \\
\quad \text { aadA1; catA1; } \operatorname{mdf}(A) ; \operatorname{tet}(A)\end{array}$ & $\begin{array}{c}\text { ast } A, \text { eae, esp } A, \operatorname{esp} B, \text { gad, iss, } \\
\text { mchF, nle A, tccP, tir }\end{array}$ \\
\hline LREC-122 & O153:H10 & A & $11-54$ & 10 & $\begin{array}{c}\text { IncF (F2:A-:B-) } \\
\text { IncI1 (STunknown) } \\
\text { IncX1 } \\
\text { Col156 } \\
\text { Col (MG828) }\end{array}$ & $\begin{array}{c}\text { bla }_{C T X-M-32} ; \operatorname{aac}(3)-I I a, \text { aadA1; cat } A 1 \\
m d f(A) ; \operatorname{tet}(A)\end{array}$ & $\begin{array}{c}\text { ast } A, \text { eae, esp } A, \operatorname{esp} B, \text { gad, iss, } \\
m c h F, \text { nle } A, \text { tccP, tir }\end{array}$ \\
\hline LREC-123 & O153:H10 & A & $11-54$ & 10 & $\begin{array}{c}\text { IncF (F2:A-:B-) } \\
\text { IncI1 (STunknown) } \\
\text { IncX1 } \\
\text { Col156 } \\
\text { Col (MG828) }\end{array}$ & $\begin{array}{l}\text { bla }_{C T X-M-32}, b l a_{T E M-1 A} ; a a c(3)-I I a, \\
\quad \text { aadA1; } \operatorname{cat} A 1 ; \operatorname{mdf}(A) ; \operatorname{tet}(A)\end{array}$ & $\begin{array}{c}\text { ast } A, \text { eae, esp } A, \operatorname{esp} B, \text { gad, iss, } \\
\text { mchF, nle A, tccP, tir }\end{array}$ \\
\hline
\end{tabular}


Table 2. Cont.

\begin{tabular}{|c|c|c|c|c|c|c|c|}
\hline Code & Serotype $^{1}$ & Phylo Group ${ }^{2}$ & $\underset{3}{\text { CHType }}$ & $\mathrm{ST}^{4}$ & $\begin{array}{l}\text { Plasmid Content Inc } \\
\text { Group (pMLST) }\end{array}$ & $\begin{array}{l}\text { Acquired Resistances (black) and } \\
\text { Point Mutations (blue) }{ }^{6}\end{array}$ & Virulence Genes 7,8 \\
\hline LREC-124 & O153:H10 & A & $11-54$ & 10 & $\begin{array}{c}\text { IncF (F2:A-:B-) } \\
\text { IncI1 (STunknown) } \\
\text { IncX1 } \\
\text { IncY } \\
\text { Col156 }\end{array}$ & $\begin{array}{l}\text { bla } a_{C T X-M-32}, b l a_{T E M-1 A} ; a a c(3)-I I a, \\
\quad \text { aadA1; } \operatorname{cat} A 1 ; \operatorname{mdf}(A) ; \operatorname{tet}(A)\end{array}$ & $\begin{array}{l}\text { ast } A, \text { eae, esp } A, \operatorname{esp} B, \operatorname{esp} F \\
\text { gad, iss, } m c h F, \text { tccP, tir }\end{array}$ \\
\hline LREC-125 & O153:H10 & A & $11-54$ & 10 & $\begin{array}{c}\text { IncF (F2:A-:B-) } \\
\text { IncI1 (STunknown) } \\
\text { IncX1 } \\
\text { Col156 }\end{array}$ & $\begin{array}{c}\operatorname{bla}_{C T X-M-32} ; \operatorname{aad} A 1 ; \operatorname{cat} A 1 ; \operatorname{mdf}(A) \\
\operatorname{tet}(A)\end{array}$ & $\begin{array}{l}\text { ast } A, \text { eae, } \operatorname{esp} A, \operatorname{esp} B, \operatorname{espF}, \\
\text { gad, iss, mchF, nleA, tccP, tir }\end{array}$ \\
\hline LREC-127 & O153:H10 & A & $11-54$ & 10 & $\begin{array}{c}\text { IncF (F2:A-:B-) } \\
\text { IncI1 (STunknown) } \\
\text { IncX1 } \\
\text { Col156 } \\
\text { Col (MG828) }\end{array}$ & $\begin{array}{l}\text { bla }_{C T X-M-32}, b l a_{T E M-1 A} ; a a c(3)-I I a \\
\quad \text { aadA1; } \operatorname{cat} A 1 ; \operatorname{mdf}(A) ; \operatorname{tet}(A)\end{array}$ & $\begin{array}{l}\text { ast } A, \text { eae, } \operatorname{esp} A, \operatorname{esp} B, \operatorname{espF}, \\
\text { gad, iss, mchF, nle A, tccP, tir }\end{array}$ \\
\hline
\end{tabular}

${ }^{1}$ Serotypes, ${ }^{3}$ clonotypes, ${ }^{4}$ sequence types, ${ }^{5}$ replicon/plasmid STs, ${ }^{6}$ acquired antimicrobial resistance genes and/or chromosomal mutations, ${ }^{7}$ virulence genes were determined using SerotypeFinder 2.0, CHtyper 1.0, MLST 2.0, PlasmidFinder 2.0, pMLST 2.0, ResFinder 3.1 and VirulenceFinder 2.0 online tools at the CGE, respectively. While ${ }^{2}$ phylogroups were predicted using the ClermonTyping tool at the Iame-research Center web. ${ }^{1}$ Serotypes: underlined and in red those (LREC-121, LREC-120) that were not predicted (ONT) by SerotypeFinder but assigned as $\mathrm{O} 153$ by conventional typing. ${ }^{6}$ Resistome: Acquired resistance genes: beta-lactam: bla $a_{\mathrm{TEM}-1 \mathrm{~A}}$, bla $a_{\mathrm{CTX}-\mathrm{M}-32}$, bla $a_{\mathrm{SHV}-12}$; aminoglycosides: aac (3)-IIa, aadA1, aadA2; phenicols: catA1, cmlA1; macrolides: $m d f(A)$; sulphonamides: sul3; tetracycline: $\operatorname{tet}(A)$. Point mutations (marked in blue): quinolones and fluoroquinolones: gyrA S83L: TCG-TTG. Underlined and in red those bla $_{\mathrm{CTX}-\mathrm{M}-32}$ genes (LREC-112, LREC-119) that were not predicted by ResFinder but determined in conventional typing ${ }^{8}$ Virulence genes: astA: EAST-1, eae: intimin, espA: type III secretions system, espB: secreted protein B, espF: type III secretions system, gad: glutamate descarboxylase, iss: increased serum survival, $m c h F$ : ABC transporter protein MchF, nleA: non-LEE-encoded effector A, tccP: Tir cytoskeleton coupling protein, tir: translocated intimin receptor protein. bp: base pairs; CHType: clonotype (fumC-fimH); ST: sequence type according to Achtman scheme; pMLST: plasmid sequence type. 
Table 3. Asymmetric distance matrix based on the cgMLST scheme from EnteroBase in which D (a, b) equals all sites that are present in (b) and different from (a).

\begin{tabular}{|c|c|c|c|c|c|c|c|c|c|c|c|c|c|c|c|c|c|c|}
\hline \multirow{2}{*}{\multicolumn{2}{|c|}{$\begin{array}{l}\text { Genome Code/ } \\
\text { cgMLST }\end{array}$}} & \multirow{2}{*}{$\begin{array}{c}\begin{array}{c}\text { LREC- } \\
110\end{array} \\
37600 \\
\end{array}$} & \multirow{2}{*}{$\begin{array}{c}\begin{array}{c}\text { LREC- } \\
111\end{array} \\
37601\end{array}$} & \multirow{2}{*}{$\begin{array}{c}\begin{array}{c}\text { LREC- } \\
127\end{array} \\
37602\end{array}$} & \multirow{2}{*}{$\begin{array}{c}\text { LREC- } \\
112 \\
37605 \\
\end{array}$} & \multirow{2}{*}{$\begin{array}{c}\begin{array}{c}\text { LREC- } \\
113\end{array} \\
37606\end{array}$} & \multirow{2}{*}{$\begin{array}{c}\begin{array}{c}\text { LREC- } \\
120\end{array} \\
37607 \\
\end{array}$} & \multirow{2}{*}{$\begin{array}{c}\begin{array}{c}\text { LREC- } \\
117\end{array} \\
37609 \\
\end{array}$} & \multirow{2}{*}{$\begin{array}{c}\text { LREC- } \\
121 \\
37610\end{array}$} & \multirow{2}{*}{$\begin{array}{c}\text { LREC- } \\
119 \\
37611\end{array}$} & \multirow{2}{*}{$\begin{array}{c}\text { LREC- } \\
116 \\
37612\end{array}$} & \multirow{2}{*}{$\begin{array}{c}\text { LREC- } \\
115 \\
37613\end{array}$} & \multirow{2}{*}{$\begin{array}{c}\begin{array}{c}\text { LREC- } \\
114\end{array} \\
37614\end{array}$} & \multirow{2}{*}{$\begin{array}{c}\begin{array}{c}\text { LREC- } \\
123\end{array} \\
37615\end{array}$} & \multirow{2}{*}{$\begin{array}{c}\begin{array}{c}\text { LREC- } \\
122\end{array} \\
37616\end{array}$} & \multirow{2}{*}{$\begin{array}{c}\text { LREC- } \\
124 \\
37617\end{array}$} & \multirow{2}{*}{$\begin{array}{c}\text { LREC- } \\
125 \\
37618\end{array}$} & \multirow{2}{*}{$\begin{array}{c}\text { LREC- } \\
118 \\
38299\end{array}$} \\
\hline & & & & & & & & & & & & & & & & & & \\
\hline LREC-110 & 37600 & 0 & 14 & 17 & 19 & 13 & 12 & 18 & 19 & 12 & 15 & 27 & 8 & 15 & 15 & 18 & 16 & 14 \\
\hline LREC-111 & 37601 & 14 & 0 & 21 & 23 & 16 & 20 & 22 & 23 & 16 & 19 & 30 & 11 & 19 & 18 & 22 & 20 & 17 \\
\hline LREC-127 & 37602 & 17 & 21 & 0 & 24 & 9 & 22 & 14 & 16 & 9 & 11 & 24 & 15 & 13 & 13 & 15 & 13 & 13 \\
\hline LREC-112 & 37605 & 19 & 23 & 24 & 0 & 19 & 25 & 24 & 25 & 18 & 21 & 33 & 17 & 22 & 22 & 24 & 23 & 21 \\
\hline LREC-113 & 37606 & 13 & 16 & 9 & 19 & 0 & 18 & 9 & 11 & 5 & 6 & 18 & 10 & 9 & 8 & 11 & 9 & 8 \\
\hline LREC-120 & 37607 & 12 & 20 & 22 & 25 & 18 & 0 & 23 & 23 & 18 & 20 & 33 & 14 & 20 & 20 & 24 & 22 & 19 \\
\hline LREC-117 & 37609 & 18 & 22 & 14 & 24 & 9 & 23 & 0 & 17 & 8 & 12 & 22 & 15 & 14 & 13 & 14 & 12 & 13 \\
\hline LREC-121 & 37610 & 19 & 23 & 16 & 25 & 11 & 23 & 17 & 0 & 10 & 13 & 25 & 15 & 16 & 16 & 16 & 14 & 14 \\
\hline LREC-119 & 37611 & 12 & 16 & 9 & 18 & 5 & 18 & 8 & 10 & 0 & 6 & 17 & 10 & 9 & 9 & 7 & 6 & 8 \\
\hline LREC-116 & 37612 & 15 & 19 & 11 & 21 & 6 & 20 & 12 & 13 & 6 & 0 & 22 & 12 & 11 & 11 & 13 & 11 & 10 \\
\hline LREC-115 & 37613 & 27 & 30 & 24 & 33 & 18 & 33 & 22 & 25 & 17 & 22 & 0 & 22 & 24 & 23 & 23 & 15 & 20 \\
\hline LREC-114 & 37614 & 8 & 11 & 15 & 17 & 10 & 14 & 15 & 15 & 10 & 12 & 22 & 0 & 13 & 12 & 15 & 14 & 11 \\
\hline LREC-123 & 37615 & 15 & 19 & 13 & 22 & 9 & 20 & 14 & 16 & 9 & 11 & 24 & 13 & 0 & 7 & 15 & 13 & 8 \\
\hline LREC-122 & 37616 & 15 & 18 & 13 & 22 & 8 & 20 & 13 & 16 & 9 & 11 & 23 & 12 & 7 & 0 & 15 & 13 & 9 \\
\hline LREC-124 & 37617 & 18 & 22 & 15 & 24 & 11 & 24 & 14 & 16 & 7 & 13 & 23 & 15 & 15 & 15 & 0 & 12 & 14 \\
\hline LREC-125 & 37618 & 16 & 20 & 13 & 23 & 9 & 22 & 12 & 14 & 6 & 11 & 15 & 14 & 13 & 13 & 12 & 0 & 12 \\
\hline LREC-118 & 38299 & 14 & 17 & 13 & 21 & 8 & 19 & 13 & 14 & 8 & 10 & 20 & 11 & 8 & 9 & 14 & 12 & 0 \\
\hline
\end{tabular}




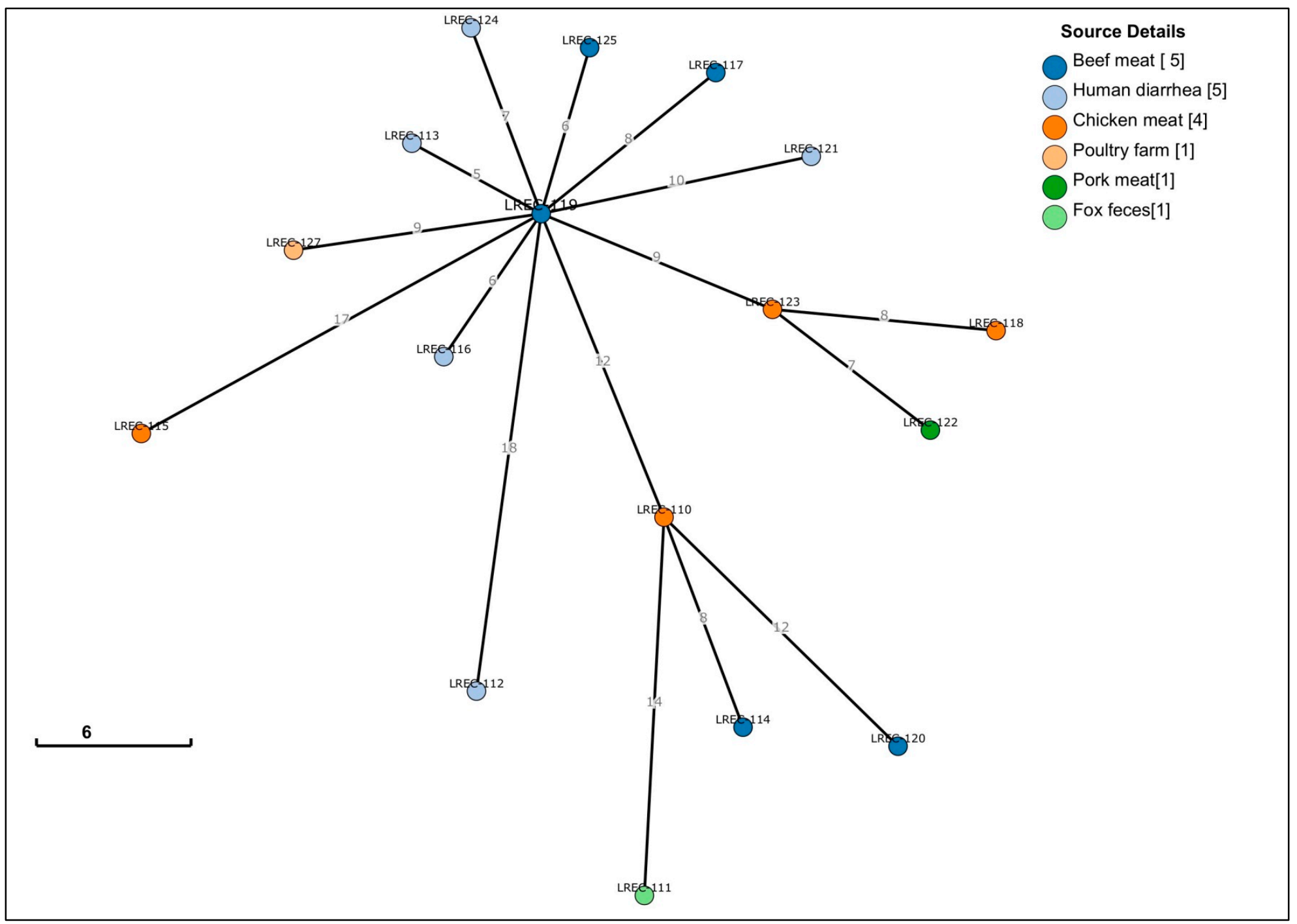

Figure 2. GrapeTree inferred using the MSTree V2 algorithm based on the cgMLST V1 + Hierarchical Clustering (HierCC) V1 scheme from EnteroBase. 


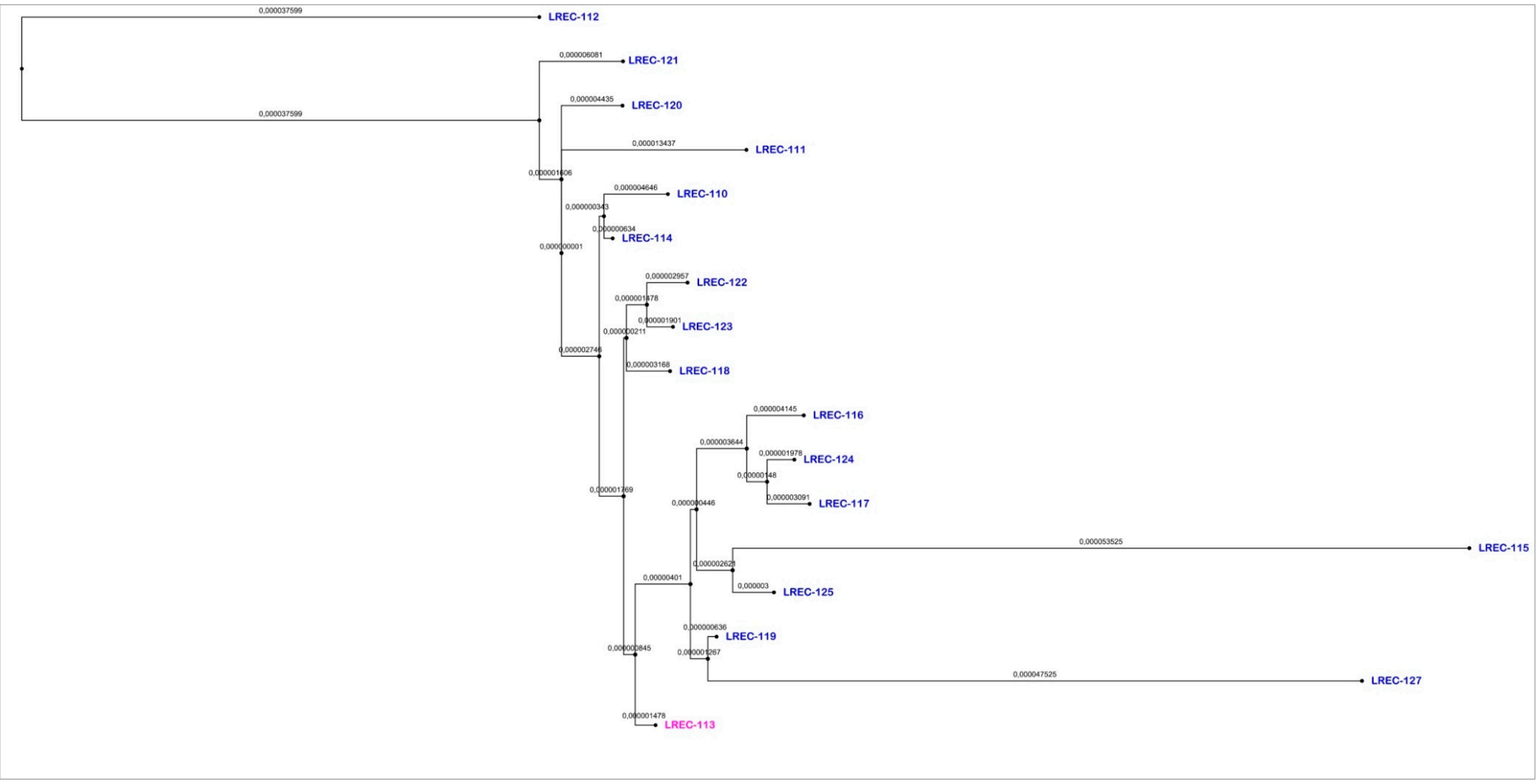

Figure 3. Dendrogram based on the SNPs of the core genomic regions present in $90 \%$ of the compared genomes and using LREC-113 as reference, built in EnteroBase and modified with FigTree v1.4.3. 


\section{Discussion}

The recovery from different sources of eae-positive E. coli isolates of serotype O153:H10, and its association with ESBL enzymes triggered this investigation. In independent studies on ESBLs in our region, we found that O153 aEPEC represented 5.5\% of the ESBL-producing E. coli recovered from chicken meat (2009-2010), 7.7\% of pork meat (2011-2012), 20\% of beef meat (2011-2012), 1\% of poultry farm environments (2010-2012) and 1\% of wildlife feces (2014-2015) [22]. In addition, we detected 23 $(0.24 \%)$ O153 aEPEC as the only pathogen within 9,523 stools of epidemiologically unrelated patients (2006-2012) in the routine testing of human diarrheagenic samples. From those 23, $14(0.15 \%)$ were

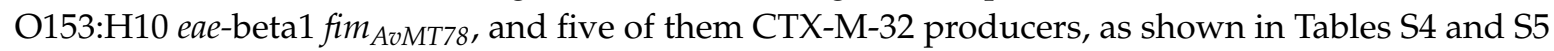
and Figure 1. By conventional typing, all animal and human isolates were assigned to the clonal group O153:H10-A-ST10 (CH11-54), conforming a hybrid aEPEC/ExPEC pathotype not previously described. The symptomatology reported in humans was mainly mild diarrhea, but there were also some cases of acute and hemorrhagic gastroenteritis, as shown in Table S5. Epidemiological studies have indicated that aEPEC are emerging enteropathogens, implicated in human diarrhea, with higher prevalence than tEPEC in both developed and developing countries [23]. aEPEC are present in both healthy and diseased animals and humans $[8,24,25]$, are phylogenetically heterogeneous, and carry virulence factors of other diarrheagenic E. coli more often than tEPEC strains $[6,23,26]$. However, the main feature of the EPEC diarrheagenic group is the ability to induce A/E lesions on intestinal epithelium encoded in the chromosomal pathogenicity island LEE. Within more than 30 intimin types and subtypes based on the polymorphism of eae, the subtype determined here (beta-1) is first or second in prevalence within different studies on isolates from humans with diarrhea in Spain [24], Australia [27], Brazil [28,29], Peru [30] or China [31].

It is of note that in subsequent and current studies, we have detected this clonal group on meat samples from supermarkets in our city. In fact, we recovered aEPEC/ExPEC from 15 out of 100 poultry meat samples (2016-2017), and of those, five were carriers of isolates belonging to the clonal group O153:H10-A-ST10, including one CTX-M-32 producer (unpublished data). Recently, Zhang et al. [32] reported a $2.75 \%$ prevalence of aEPEC in retail foods at markets in the People's Republic of China, with the beta-1 intimin and the ST10, as the second intimin and ST most prevalent within their isolates. According to the authors, the presence of virulent and MDR aEPEC in retail foods poses a potential threat to consumers. While O153 is a serogroup reported within ExPEC and DEC isolates, and linked to different lineages [33-36], there are few references of the serotype O153:H10. Notheworthy, the report by Schremmer et al [37] of an aEPEC O153:H10 isolated from the small intestine of a cockatiel (psittaciform bird) with enteritis.

Since the occurrence of the major outbreak of HUS in Europe in 2011 caused by an EAggEC/STEC O104:H4, other hybrid pathotypes have been recognized and new ones are expected, either by novel assemblies of $E$. coli virulence determinants or through the acquisition of new virulence genes from other bacterial species [16]. In Norway, Lindstedt et al. [38], expressed their concern regarding the detection of E. coli from human fecal content with a combination of intestinal and ExPEC virulence genes (IPEC/ExPEC) in a high frequency (64.3\%). Several other studies have also identified STEC- and ETEC-associated virulence genes coexisting in E. coli isolates from humans, animals or environmental origin $[39,40]$. However, one of the most outstanding is the EPEC/STEC O80:H2-ST301, which emerged in France over the last few years and diffused within Europe. This emerging hybrid is associated with invasive infections, and combines intestinal VFs (st $x 2 d$, eae-xi and ehxA genes) and extraintestinal genes characteristic of the plasmid pS88 [41,42]. In this O80 clone, it is to highlight the location of MDR and pS88 genes in the same plasmid, as well as the presence of two additional plasmids (a carrier of ehxA gene and a cryptic one) within the isolates [41,42]. The clonal group described here poses also the threat of being MDR and characteristically associated with ESBL-type CTX-M-32. The CTX-M-32 enzyme is derived from CTX-M-1 by a single amino acid replacement, probably an ancestor among CTX-M-1 and CTX-M-15 [43]. The bla CTX-M-32 gene was first described in 2004 in an Escherichia coli isolate in our Health Area (A Coruña, northwest Spain) [43]. Furthermore, it was described in three 
human isolates O25b:H4-ST131 ibeA-positive of our region, as early as in 2008 [14]. Of the 2,427 E. coli bloodstream isolates recovered in the hospital of our city (HULA) in the period 2000-2011, 96 were positive for ESBL production, from which 4.2\% were CTX-M-32 and 4.2\% SHV-12 [44]. The same prevalence was observed in this hospital in 2015 (unpublished data).

The in silico analysis of 17 representative genomes O153:H10-A-ST10 corroborated the main traits determined by conventional typing. In a recent study, we proved the good correlation and usefulness of SerotypeFinder or EnteroBase predictions [25,45]. Here, only the serotype of two genomes could not be predicted in silico, probably due to the limitation of the assembly based on Illumina short reads [46]. MLST, CHTyper from CGE and EnteroBase also confirmed conventional results. Like in the previous study, we found that VirulenceFinder verified the E. coli pathotypes (aEPEC/ExPEC) established by PCR. However, VirulenceFinder identified different traits for the ExPEC pathotype. Thus, this clonal group O153:H10-A-ST10 typically carries the locus encoding a fimA variant MT78 of type 1 fimbriae [19], and the traT gene for an outer membrane protein implicated in serum survival [20]. Both VFs are not included in the VirulenceFinder scheme and so, they are not predicted. On the contrary, the CGE tool allowed the identification of the increased serum survival gene iss, recognized for its role in ExPEC virulence [21], in all genomes. The CGE database predicts 14 variants of the iss gene [47], including the one described in E. coli IAI1 (CU928160), and harbored by the O153:H10-A-ST10 genomes. Our specific PCR detects the plasmid-borne iss allele (designated type 1), which is highly prevalent among avian pathogenic $E$. coli and neonatal meningitis-associated $E$. coli isolates, but not among uropathogenic E. coli isolates [21]. The phenotypic AMR determined in vitro correlated with the results predicted by ResFinder databases, with the exception of the $b l a_{\mathrm{CTX}-\mathrm{M}-32}$ gene not predicted in two genomes, but solved by PCR and sequencing. Based on this, and previous studies [45,48], we consider both conventional and genomic-based analysis complementary for a better understanding and characterization of emerging isolates.

An important trait found in this study was the concomitant presence of IncF (F2:A-:B-), IncI1 (STunknown) and IncX1, together with non-conjugative Col156-like plasmids in 15 of the 17 genomes. Although carriage of plasmids places a fitness burden on their host [49], different studies support the hypothesis that interference between conjugative plasmids may reduce fitness cost by decreasing the efficiency of transfer. However, the mechanisms of such inhibitory systems need further investigation [50]. On the other hand, small plasmids were shown to increase their stability in cells containing big plasmids [49]. We hypothesized here that the stable combination of IncF (F2:A-:B-), IncI1 (STunknown) and IncX1 plasmid types, together with non-conjugative Col-like types might be implicated in the successful persistence of this hybrid pathotype, and the spread of antibiotic resistance genes.

Another objective in this study was to know if this was a geographically restricted genetic lineage, or if it had been reported somewhere else. For this purpose, and based on the HierCC Cluster ID, we searched related genomes uploaded in EnteroBase. As a result, we found a hybrid aEPEC/ExPEC pathotype A-ST10 eae-beta1 on its database associated to five humans, one avian, and one unknown isolates, as shown in Table S3. Of note are the two human O153:H10-A-ST10 (CH11-54) eae-beta1 isolates (Code Name: 853984 and 866428) from the United Kingdom, which clustered with the 17 Spanish genomes in the HC100 HierCC group (37600), as shown in Table S3 and Figure S1. Additionally, the in silico analysis of these two genomes showed that they were MDR, and carried similar virulence traits (conforming hybrid aEPEC/ExPEC pathotype) and plasmid combination: IncF (F2:A-:B-), IncX1 and Col156-like, as shown in Table S6. Importantly, six of the seven hybrid aEPEC/ExPEC genomes found in EnteroBase were carriers of IncF (F2:A-:B-) and Col156-like plasmids, as shown in Table S6. As above suggested, further investigation on the interplay between these plasmids and other mobile genetic elements affecting their transmission and persistence, as well as their role in the maintenance and acquisition of resistance genes is necessary. 


\section{Materials and Methods}

\subsection{E. coli Collection}

From 2005 to 2015, different surveillance studies performed at the Reference Laboratory of Escherichia coli (LREC), in Lugo, Spain, aimed to detect ESBL-producing E. coli within different sources of our region. These studies included samples from chicken, beef and pork meat, as well as poultry farm environments (avian feces taken from the floor) and wildlife (fox feces). Briefly, the confluent growth of the MacConkey Lactose plates from each sample was screened by PCR for the presence of specific bla genes using the TEM, CIT, SHV, CTX-M-1 and CTX-M-9 group-specific primers [51]. Then, up to ten individual colonies from positive plates were reanalyzed. Those confirmed for the bla genes were further characterized by PCR for the presence of VF eae, st 1 , st $x 2$, ipaH, pcDV432, elt $A$, est $A$ or $e s t B$ associated with the main intestinal pathotypes (enteropathogenic, verotoxigenic, enteroinvasive, enteroaggregative and enterotoxigenic) of $E$. coli. Likewise, specific extraintestinal VF were tested: fimH, fimAv $v_{\mathrm{MT} 78}$, papC, sfa/focDE, afa/draBC, cnf1, cdtB, sat, hlyA, iucD, iroN, kpsM II (establishing neuC-K1, $\mathrm{K} 2$ and $\mathrm{K} 5$ variants), kpsM III, cvaC, iss, traT, ibeA, malX, usp and tsh (Tables S7-S9).

On the other hand, human diarrheagenic E. coli isolates, mainly from the Hospital Universitario Lucus Augusti (HULA) of our city (Lugo, northwest Spain), were routinely analyzed in our laboratory for intestinal VF, and those positive, complementary analyzed for extraintestinal traits and ESBL genes, as described in the preceding paragraph.

All isolates were serotyped using the method previously described by Guinée et al. [52] employing O1 to O185 and H1 to H56 antisera. As a result, 32 eae-positive E. coli (21 ESBL and 11 non-ESBL) belonging to the serotype O153:H10 constituted the collection of the study, as shown in Table S1.

\subsection{Antimicrobial Susceptibility and ESBL Typing}

Antimicrobial susceptibility testing was conducted by disk (Becton Dickinson, Sparks, MD, USA) diffusion assay. The antibiotics tested included ampicillin (AMP), amoxicillin/clavulanic acid (AMC), cefuroxime (CXM), ceftazidime (CAZ), cefotaxime (CTX), cefepime (FEP), cefoxitin (FOX), aztreonam (ATM), imipenem (IMP), gentamicin (GEN), tobramycin (TOB), fosfomycin (FOF), nitrofurantoin (NIT), sulfamethoxazole/trimethoprim (SXT), ciprofloxacin (CIP) and nalidixic acid (NAL). The assays were performed, and all results interpreted, according to the CLSI guidelines [53]. The isolates were investigated by PCR for screening of specific bla genes using the TEM, SHV, CTX-M-1 and CTX-M-9 group-specific primers (Table S9), and further sequencing as described elsewhere [51].

\subsection{Phylogenetic Assignment and PFGE Comparison}

Phylogroup and ST assignment was performed following the Clermont et al. [54] and Achtman MLST [55] schemes, respectively. The clonotyping was based on the internal 469-nucleotide (nt) and 489-nt sequence of the fumC and fimH genes, respectively, to define the $\mathrm{CH}$ type [56]. The molecular similarity within the collection was established by comparing the XbaI-PFGE profiles of the isolates obtained following the PulseNet protocol, and imported into BioNumerics (Applied Maths, St-Martens-Latem, Belgium) to perform a dendrogram with the UPGMA algorithm based on the Dice similarity coefficient and applying $1 \%$ of tolerance in the band position.

\subsection{Genome Sequencing, Assembly and Analysis}

DNA from 17 isolates was extracted with the QIAamp 96 DNA Qiacube HT kit (Qiagen, Hilden, Germany) and libraries were prepared using the Nextera XT kit (Illumina). Pooled libraries were denatured following the Illumina protocol and $600 \mu \mathrm{l}$ (approx. $20 \mathrm{pM}$ ) were loaded onto a MisSeq V2 -500 cycle cartridge (Illumina) and sequenced on a MiSeq to produces fastq files. Raw reads were uploaded and automatically assembled in EnteroBase using SPAdes Genome Assembler v 3.5. with a contig threshold of minimum 200 nucleotides. Subsequently, the de novo assembled contigs were MLST (7 gene Achtman ST scheme, whole genome MLST, core genome MLST and ribosomal MLST) 
and serotyped in silico using EnteroBase typing tools [57]. The raw reads were also analyzed using the following CGE databases: SerotypeFinder, MLSTtyper, CHTyper, PlasmidFinder, ResFinder, and VirulenceFinder [58-62]. For genomic relatedness comparison, we used different approaches based on the cgMLST of EnteroBase. Thus, a MSTree was inferred using the MSTree V2 algorithm and the asymmetric distance matrix based on the cgMLST scheme from EnteroBase. This cgMLST scheme consists of 2,513 genes present in over $98 \%$ of 3,457 genomes, which represented most of the diversity in EnteroBase https:/enterobase.readthedocs.io/en/latest/pipelines/escherichia-statistics.html. We also investigated the HierCC designations for our collection and other related genomes of EnteroBase within each cluster group $[57,63]$. The SNP tree was also built in EnteroBase, where all assemblies were aligned against LREC-113 using Last [64], and SNPs from these alignments were filtered to remove regions with low base qualities or ambiguous alignment. Specifically, any sites with low base qualities $(\mathrm{Q}<10)$ or sites which could not be aligned unambiguously (ambiguity of alignment $\geq 0.1$, as reported by Last) were excluded. Additionally sites were removed if disperse repetitive regions were aligned with $\geq 95 \%$ identities and longer than $\geq 100$ bps according to nucleotide BLAST; if they were part of tandem repeats that were identified by TRF [65]; if they were within CRISPR regions, which were identified by PILER-CR [66]. After removing repetitive regions, all core SNPs were then called in the core genomic regions that were conserved in $\geq 90 \%$ of the genomes.

\section{Conclusions}

In summary, our results demonstrate that a hybrid MDR aEPEC/ExPEC belonging to the clonal group O153:H10-A-ST10 (CH11-54) eae-beta1 is circulating in our region within different hosts, including wildlife. It seems implicated in human diarrhea via food (meat) transmission, and in the spreading of ESBL genes (mainly of CTX-M-32 type). The concomitant presence of IncF (F2:A-:B-), IncI1 (STunknown) and IncX1, together with non-conjugative Col156-like plasmids might be implicated in the successful persistence of this hybrid pathotype. We found genomic evidence of a related hybrid aEPEC/ExPEC in at least one other country.

\section{Data Availability}

The whole genome sequenced samples are part of BioProject PRJEB19190 and correspond to BioSample IDs: SAMEA92137918 (LREC-110); SAMEA92139418 (LREC-111); SAMEA92142418 (LREC-112), SAMEA92143168 (LREC-113); SAMEA92149918 (LREC-114); SAMEA92149168 (LREC-115); SAMEA92148418 (LREC-116); SAMEA92146168 (LREC-117); SAMEA92154418 (LREC-118); SAMEA 92147668 (LREC-119); SAMEA92144668 (LREC-120); SAMEA92146918 (LREC-121); SAMEA92151418 (LREC-122); SAMEA92150668 (LREC-123); SAMEA92152168 (LREC-124); SAMEA92152918 (LREC-125); SAMEA92140168 (LREC-127).

Supplementary Materials: The following are available online at http://www.mdpi.com/2079-6382/9/4/192/s1, Table S1: Thirty-two isolates included in the study from our own collections, Table S2: Assembly data from EnteroBase of the 17 O153:H10-A-ST10 genomes sequenced using Illumina NextSeq technology, Table S3: HierCC designations from EnteroBase for the 17 Spanish collection and other 7 related genomes within each cluster group. SNPs of the core genomic regions, Table S4: Number of human stool samples analyzed and positive for aEPEC O153, Table S5: Twenty-three aEPEC O153 human isolates recovered in the period 2006-2012, Table S6: in silico characterization of seven E. coli related genomes from EnteroBase using CGE databases, Table S7: Targets and primers associated with diarrheagenic pathotypes of E. coli, Table S8: Targets and primers associated with extraintestinal pathotypes of E. coli, Table S9: Detection and sequencing of bla $a_{\mathrm{TEM}}, b l a_{\mathrm{SHV}}$ and $b l a_{\mathrm{CTX}-\mathrm{M}}$ genes, Figure S1: GrapeTree inferred using the NINJA NJ algorithm and based on the cgMLST V1 + HierCC V1 scheme from EnteroBase.

Author Contributions: Conceptualization, A.M.; methodology, A.M., J.B., A.H. and A.M.L.-B.; formal analysis, D.D.-J., I.G.-M., A.H., M.P.A., A.M.L.-B. and V.G.; investigation, A.M. and J.B.; writing-original draft preparation, A.M.; writing-review and editing, A.M., I.G.-M., D.D.-J., and V.G.; supervision, A.M.; funding acquisition, A.M. and J.B. All authors have read and agreed to the published version of the manuscript.

Funding: This study was supported by projects: AGL2013-47852-R from the Ministerio de Economía y Competitividad (MINECO, Spain) and Fondo Europeo de Desarrollo Regional (FEDER); AGL2016-79343-R from the Agencia Estatal de Investigación (AEI, Spain) and FEDER; PI16/01477 from Plan Estatal de I+D+I 
2013-2016, Instituto de Salud Carlos III (ISCIII), Subdirección General de Evaluación y Fomento de la Investigación and FEDER; and ED431C2017/57 from the Consellería de Cultura, Educación e Ordenación Universitaria of Xunta de Galicia and FEDER.

Acknowledgments: I.G.-M., D.D.-J. and V.G. acknowledge the Consellería de Cultura, Educación e Ordenación Universitaria, Xunta de Galicia for their pre-doctoral and post-doctoral grants (Grant Numbers ED481A-2015/149, ED481A-2019/022 and ED481B-2018/018, respectively). A. Mora acknowledges the Ministerio de Educación, Cultura y Deporte (Spain) for the mobility grant PRX16/00023 for teachers and researchers from the Programa Estatal de Promoción del Talento y su Empleabilidad, Plan Estatal de Investigación Científica y Técnica y de Innovación 2013-2016. She also expresses her gratitude to Mark Achtman and his group (Zhou, Sergeant and Nabil-Fareed) for their invaluable help with EnteroBase during the stay at the Microbiology and Infection Unit, Warwick Medical School, University of Warwick, Coventry, United Kingdom.

Conflicts of Interest: The authors declare no conflict of interest.

\section{References}

1. Kaper, J.B.; Nataro, J.P.; Mobley, H.L. Pathogenic Escherichia coli. Nat. Rev. Microbiol. 2004, 2, $123-140$. [CrossRef]

2. Riley, L.W. Pandemic lineages of extraintestinal pathogenic Escherichia coli. Clin. Microbiol. Infect. 2014, 20, 380-390. [CrossRef] [PubMed]

3. Caron, E.; Crepin, V.F.; Simpson, N.; Knutton, S.; Garmendia, J.; Frankel, G. Subversion of actin dynamics by EPEC and EHEC. Curr. Opin. Microbiol. 2006, 9, 40-45. [CrossRef] [PubMed]

4. Mora, A.; Blanco, M.; Yamamoto, D.; Dahbi, G.; Blanco, J.E.; Lopez, C.; Alonso, M.P.; Vieira, M.A.; Hernandes, R.T.; Abe, C.M.; et al. HeLa-cell adherence patterns and actin aggregation of enteropathogenic Escherichia coli (EPEC) and Shiga-toxin-producing E. coli (STEC) strains carrying different eae and tir alleles. Int. Microbiol. 2009, 12, 243-251. [PubMed]

5. Blanco, M.; Blanco, J.E.; Mora, A.; Dahbi, G.; Alonso, M.P.; Gonzalez, E.A.; Bernardez, M.I.; Blanco, J. Serotypes, virulence genes, and intimin types of Shiga toxin (verotoxin)-producing Escherichia coli isolates from cattle in Spain and identification of a new intimin variant gene (eae-xi). J. Clin. Microbiol. 2004, 42, 645-651. [CrossRef] [PubMed]

6. Hernandes, R.T.; Elias, W.P.; Vieira, M.A.M.; Gomes, T.A.T. An overview of atypical enteropathogenic Escherichia coli. FEMS Microbiol. Lett. 2009, 297, 137-149. [CrossRef] [PubMed]

7. Otero, V.; Rodríguez-Calleja, J.M.; Otero, A.; García-López, M.L.; Santos, J.A. Genetic characterization of atypical enteropathogenic Escherichia coli isolates from ewes' milk, sheep farm environments, and humans by multilocus sequence typing and pulsed-field gel electrophoresis. Appl. Environ. Microbiol. 2013, 79, 5864-5869. [CrossRef]

8. Alonso, C.A.; Mora, A.; Diaz, D.; Blanco, M.; Gonzalez-Barrio, D.; Ruiz-Fons, F.; Simon, C.; Blanco, J.; Torres, C. Occurrence and characterization of stx and/or eae-positive Escherichia coli isolated from wildlife, including a typical EPEC strain from a wild boar. Vet. Microbiol. 2017, 207, 69-73. [CrossRef]

9. Johnson, J.R.; Murray, A.C.; Gajewski, A.; Sullivan, M.; Snippes, P.; Kuskowski, M.A.; Smith, K.E. Isolation and molecular characterization of nalidixic acid-resistant extraintestinal pathogenic Escherichia coli from retail chicken products. Antimicrob. Agents Chemother. 2003, 47, 2161-2168. [CrossRef]

10. Spurbeck, R.R.; Dinh, P.C., Jr.; Walk, S.T.; Stapleton, A.E.; Hooton, T.M.; Nolan, L.K.; Kim, K.S.; Johnson, J.R.; Mobley, H.L. Escherichia coli isolates that carry vat, fyuA, chuA, and yfcV efficiently colonize the urinary tract. Infect. Immun. 2012, 80, 4115-4122. [CrossRef]

11. Kohler, C.D.; Dobrindt, U. What defines extraintestinal pathogenic Escherichia coli? Int. J. Med. Microbiol. 2011, 301, 642-647. [CrossRef] [PubMed]

12. Müller, A.; Stephan, R.; Nüesch-Inderbinen, M. Distribution of virulence factors in ESBL-producing Escherichia coli isolated from the environment, livestock, food and humans. Sci. Total Environ. 2016, 541, 667-672. [CrossRef] [PubMed]

13. Hindermann, D.; Gopinath, G.; Chase, H.; Negrete, F.; Althaus, D.; Zurfluh, K.; Tall, B.D.; Stephan, R.; Nuesch-Inderbinen, M. Salmonella enterica serovar Infantis from Food and Human Infections, Switzerland, 2010-2015: Poultry-Related Multidrug Resistant Clones and an Emerging ESBL Producing Clonal Lineage. Front. Microbiol. 2017, 8, 1322. [CrossRef] [PubMed] 
14. Mora, A.; Herrera, A.; Mamani, R.; Lopez, C.; Alonso, M.P.; Blanco, J.E.; Blanco, M.; Dahbi, G.; Garcia-Garrote, F.; Pita, J.M.; et al. Recent emergence of clonal group O25b:K1:H4-B2-ST131 ibeA strains among Escherichia coli poultry isolates, including CTX-M-9-producing strains, and comparison with clinical human isolates. Appl. Env. Microbiol. 2010, 76, 6991-6997. [CrossRef]

15. Stokes, H.W.; Gillings, M.R. Gene flow, mobile genetic elements and the recruitment of antibiotic resistance genes into Gram-negative pathogens. FEMS Microbiol. Rev. 2011, 35, 790-819. [CrossRef]

16. Robins-Browne, R.M.; Holt, K.E.; Ingle, D.J.; Hocking, D.M.; Yang, J.; Tauschek, M. Are Escherichia coli Pathotypes Still Relevant in the Era of Whole-Genome Sequencing? Front. Cell Infect. Microbiol. 2016, 6, 141. [CrossRef]

17. Mora, A.; Herrrera, A.; Lopez, C.; Dahbi, G.; Mamani, R.; Pita, J.M.; Alonso, M.P.; Llovo, J.; Bernardez, M.I.; Blanco, J.E.; et al. Characteristics of the Shiga-toxin-producing enteroaggregative Escherichia coli O104:H4 German outbreak strain and of STEC strains isolated in Spain. Int. Microbiol. 2011, 14, 121-141.

18. Scheutz, F. Taxonomy Meets Public Health: The Case of Shiga Toxin-Producing Escherichia coli. Microbiol. Spectr. 2014, 2. [CrossRef]

19. Marc, D.; Dho-Moulin, M. Analysis of the fim cluster of an avian O2 strain of Escherichia coli: Serogroup-specific sites within fimA and nucleotide sequence of fimI. J. Med. Microbiol. 1996, 44, 444-452. [CrossRef]

20. Johnson, J.R.; Stell, A.L. Extended virulence genotypes of Escherichia coli strains from patients with urosepsis in relation to phylogeny and host compromise. J. Infect. Dis. 2000, 181, 261-272. [CrossRef]

21. Johnson, T.J.; Wannemuehler, Y.M.; Nolan, L.K. Evolution of the iss gene in Escherichia coli. Appl. Environ. Microbiol. 2008, 74, 2360-2369. [CrossRef] [PubMed]

22. Diaz-Jimenez, D.; Zhou, Z.; Herrera, A.; Viso, S.; Blanco, M.; Costoya, L.; Mora, A. Genomic evidence of the close relatedness of food, poultry, wildlife and human clincal isolates of ESBL-producing Escherichia coli O153:H10-A-ST10 eae-beta1. In Proceedings of the 7th Congress of European Microbiologists FEMS, Valencia, Spain, 9-13 July 2017.

23. Hu, J.; Torres, A.G. Enteropathogenic Escherichia coli: Foe or innocent bystander? Clin. Microbiol. Infect. 2015, 21, 729-734. [CrossRef] [PubMed]

24. Blanco, M.; Blanco, J.E.; Dahbi, G.; Alonso, M.P.; Mora, A.; Coira, M.A.; Madrid, C.; Juarez, A.; Bernardez, M.I.; Gonzalez, E.A.; et al. Identification of two new intimin types in atypical enteropathogenic Escherichia coli. Int. Microbiol. 2006, 9, 103-110. [PubMed]

25. Mora, A.; Garcia-Pena, F.J.; Alonso, M.P.; Pedraza-Diaz, S.; Ortega-Mora, L.M.; Garcia-Parraga, D.; Lopez, C.; Viso, S.; Dahbi, G.; Marzoa, J.; et al. Impact of human-associated Escherichia coli clonal groups in Antarctic pinnipeds: Presence of ST73, ST95, ST141 and ST131. Sci. Rep. 2018, 8, 4678. [CrossRef] [PubMed]

26. Xu, Y.; Bai, X.; Jin, Y.; Hu, B.; Wang, H.; Sun, H.; Fan, R.; Fu, S.; Xiong, Y. High prevalence of virulence genes in specific genotypes of atypical enteropathogenic Escherichia coli. Front. Cell Infect. Microbiol. 2017, 7, 109. [CrossRef] [PubMed]

27. Robins-Browne, R.M.; Bordun, A.M.; Tauschek, M.; Bennett-Wood, V.R.; Russell, J.; Oppedisano, F.; Lister, N.A.; Bettelheim, K.A.; Fairley, C.K.; Sinclair, M.I.; et al. Atypical enteropathogenic Escherichia coli: A leading cause of community-acquired gastroenteritis, Melbourne, Australia. Emerg. Infect. Dis. 2004, 10, 1797-1805. [CrossRef]

28. Abe, C.M.; Trabulsi, L.R.; Blanco, J.; Blanco, M.; Dahbi, G.; Blanco, J.E.; Mora, A.; Franzolin, M.R.; Taddei, C.R.; Martinez, M.B.; et al. Virulence features of atypical enteropathogenic Escherichia coli identified by the eae $(+)$ EAF-negative stx(-) genetic profile. Diagn. Microbiol. Infect. Dis. 2009, 64, 357-365. [CrossRef]

29. Vieira, M.A.; Dos Santos, L.F.; Dias, R.C.B.; Camargo, C.H.; Pinheiro, S.R.S.; Gomes, T.A.T.; Hernandes, R.T. Atypical enteropathogenic Escherichia coli as aetiologic agents of sporadic and outbreakassociated diarrhoea in Brazil. J. Med. Microbiol. 2016, 65, 998-1006. [CrossRef]

30. Contreras, C.A.; Ochoa, T.J.; Lacher, D.W.; DebRoy, C.; Navarro, A.; Talledo, M.; Donnenberg, M.S.; Ecker, L.; Gil, A.I.; Lanata, C.F.; et al. Allelic variability of critical virulence genes (eae, bfpA and perA) in typical and atypical enteropathogenic Escherichia coli in Peruvian children. J. Med. Microbiol. 2010, 59, 25-31. [CrossRef]

31. Xu, Y.; Bai, X.; Zhao, A.; Zhang, W.; Ba, P.; Liu, K.; Jin, Y.; Wang, H.; Guo, Q.; Sun, H.; et al. Genetic diversity of intimin gene of atypical enteropathogenic Escherichia coli isolated from human, animals and raw meats in China. PLoS ONE 2016, 11, e0152571. [CrossRef] 
32. Zhang, S.; Yang, G.; Huang, Y.; Zhang, J.; Cui, L.; Wu, Q. Prevalence and characterization of atypical enteropathogenic Escherichia coli isolated from retail foods in China. J. Food Prot. 2018, 81, 1761-1767. [CrossRef] [PubMed]

33. Balière, C.; Rincé, A.; Delannoy, S.; Fach, P.; Gourmelon, M. Molecular profiling of Shiga toxin-producing Escherichia coli and enteropathogenic E. coli strains isolated from French coastal environments. Appl. Environ. Microbiol. 2016, 82, 3913-3927. [CrossRef] [PubMed]

34. Ciesielczuk, H.; Doumith, M.; Hope, R.; Woodford, N.; Wareham, D.W. Characterization of the extra-intestinal pathogenic Escherichia coli ST131 clone among isolates recovered from urinary and bloodstream infections in the United Kingdom. J. Med. Microbiol. 2015, 64, 1496-1503. [CrossRef] [PubMed]

35. Kennedy, C.A.; Walsh, C.; Karczmarczyk, M.; O’Brien, S.; Akasheh, N.; Quirke, M.; Farrell-Ward, S.; Buckley, T.; Fogherty, U.; Kavanagh, K.; et al. Multi-drug resistant Escherichia coli in diarrhoeagenic foals: Pulsotyping, phylotyping, serotyping, antibiotic resistance and virulence profiling. Vet. Microbiol. 2018, 223, 144-152. [CrossRef] [PubMed]

36. Konishi, N.; Obata, H.; Kai, A.; Ohtsuka, K.; Nishikawa, Y.; Terajima, J.; Hara-Kudo, Y. Major vehicles and o-serogroups in foodborne enterotoxigenic Escherichia coli outbreaks in Japan, and effective detection methods of the pathogen in food associated with an outbreak. J. Food Hyg. Soc. Japan 2018, 59, 161-166. [CrossRef] [PubMed]

37. Schremmer, C.; Lohr, J.E.; Wastlhuber, U.; Kosters, J.; Ravelshofer, K.; Steinruck, H.; Wieler, L.H. Enteropathogenic Escherichia coli in Psittaciformes. Avian Pathol. 1999, 28, 349-354. [CrossRef] [PubMed]

38. Lindstedt, B.-A.; Finton, M.D.; Porcellato, D.; Brandal, L.T. High frequency of hybrid Escherichia coli strains with combined Intestinal Pathogenic Escherichia coli (IPEC) and Extraintestinal Pathogenic Escherichia coli (ExPEC) virulence factors isolated from human faecal samples. BMC Infect. Dis. 2018, 18, 544. [CrossRef] [PubMed]

39. Nyholm, O.; Heinikainen, S.; Pelkonen, S.; Hallanvuo, S.; Haukka, K.; Siitonen, A. Hybrids of Shigatoxigenic and Enterotoxigenic Escherichia coli (STEC/ETEC) Among Human and Animal Isolates in Finland. Zoonoses Public Health. 2015, 62, 518-524. [CrossRef]

40. Michelacci, V.; Maugliani, A.; Tozzoli, R.; Corteselli, G.; Chiani, P.; Minelli, F.; Gigliucci, F.; Arancia, S.; Conedera, G.; Targhetta, C.; et al. Characterization of a novel plasmid encoding F4-like fimbriae present in a Shiga-toxin producing enterotoxigenic Escherichia coli isolated during the investigation on a case of hemolytic-uremic syndrome. Int. J. Med. Microbiol. 2018, 308, 947-955. [CrossRef]

41. Cointe, A.; Birgy, A.; Bridier-Nahmias, A.; Mariani-Kurkdjian, P.; Walewski, V.; Lévy, C.; Cohen, R.; Fach, P.; Delannoy, S.; Bidet, P.; et al. Escherichia coli O80 hybrid pathotype strains producing Shiga toxin and ESBL: Molecular characterization and potential therapeutic options. J. Antimicrob. Chemother. 2020, 75, 537-542. [CrossRef]

42. Cointe, A.; Birgy, A.; Mariani-Kurkdjian, P.; Liguori, S.; Courroux, C.; Blanco, J.; Delannoy, S.; Fach, P.; Loukiadis, E.; Bidet, P.; et al. Emerging multidrug-resistant hybrid pathotype shiga toxin-producing Escherichia coli $\mathrm{O} 80$ and related strains of clonal complex 165, Europe. Emerg. Infect. Dis. 2018, 24, 2262-2269. [CrossRef] [PubMed]

43. Cartelle, M.; Del Mar Tomas, M.; Molina, F.; Moure, R.; Villanueva, R.; Bou, G. High-level resistance to ceftazidime conferred by a novel enzyme, CTX-M-32, derived from CTX-M-1 through a single Asp240-Gly substitution. Antimicrob. Agents Chemother. 2004, 48, 2308-2313. [CrossRef] [PubMed]

44. Mamani, R.; Camille Flament-Simon, S.; García Menéndez, V.; Mora, A.; Pilar Alonso, M.; López, C.; García-Meniño, I.; Díaz-Jiménez, D.; Blanco, J.; Blanco, M.; et al. Sequence Types, Clonotypes, Serotypes, and Virotypes of Extended-Spectrum $\beta$-Lactamase-Producing Escherichia coli Causing Bacteraemia in a Spanish Hospital Over a 12-Year Period (2000 to 2011). Front. Microbiol. 2019, 10. [CrossRef] [PubMed]

45. Garcia-Meniño, I.; Diaz-Jimenez, D.; Garcia, V.; de Toro, M.; Flament-Simon, S.C.; Blanco, J.; Mora, A. Genomic Characterization of Prevalent mcr-1, mcr-4, and mcr-5 Escherichia coli Within Swine Enteric Colibacillosis in Spain. Front. Microbiol. 2019, 10, 2469. [CrossRef]

46. Wick, R.R.; Judd, L.M.; Gorrie, C.L.; Holt, K.E. Completing bacterial genome assemblies with multiplex MinION sequencing. Microb. Genomics. 2017, 3. [CrossRef]

47. Joensen, K.G.; Scheutz, F.; Lund, O.; Hasman, H.; Kaas, R.S.; Nielsen, E.M.; Aarestrup, F.M. Real-time whole-genome sequencing for routine typing, surveillance, and outbreak detection of verotoxigenic Escherichia coli. J. Clin. Microbiol. 2014, 52, 1501-1510. [CrossRef] 
48. De Toro, M.; Fernandez, J.; Garcia, V.; Mora, A.; Blanco, J.; de la Cruz, F.; Rodicio, M.R. Whole genome sequencing, molecular typing and in vivo virulence of OXA-48-producing Escherichia coli isolates including ST131 H30-Rx, H22 and H41 subclones. Sci. Rep. 2017, 7, 12103. [CrossRef]

49. San Millan, A.; MacLean, R.C. Fitness Costs of Plasmids: A Limit to Plasmid Transmission. Microbiol. Spectr. 2017, 5. [CrossRef]

50. Dionisio, F.; Zilhão, R.; Gama, J.A. Interactions between plasmids and other mobile genetic elements affect their transmission and persistence. Plasmid 2019, 102, 29-36. [CrossRef]

51. Mora, A.; Viso, S.; Lopez, C.; Alonso, M.P.; Garcia-Garrote, F.; Dabhi, G.; Mamani, R.; Herrera, A.; Marzoa, J.; Blanco, M.; et al. Poultry as reservoir for extraintestinal pathogenic Escherichia coli O45:K1:H7-B2-ST95 in humans. Vet. Microbiol. 2013, 167, 506-512. [CrossRef]

52. Guinée, P.A.M.; Jansen, W.H.; Wadström, T.; Sellwood, R. Escherichia coli associated with neonatal diarrhoea in piglets and calves. In Laboratory Diagnosis in Neonatal Calf and Pig Diarrhoea: Current Topics in Veterinary and Animal Science, 13; Leeww, P.W., Guinée, P.A.M., Eds.; Springer: Dordrecht, Germany, 1981; pp. 126-162.

53. Clinical and Laboratory Standards Institute. Performance Standars for Antimicrobial Susceptibility Testing; Clinical and Laboratory Standards Institute: Wayne, PA, USA, 2019.

54. Clermont, O.; Christenson, J.K.; Denamur, E.; Gordon, D.M. The Clermont Escherichia coli phylo-typing method revisited: Improvement of specificity and detection of new phylo-groups. Env. Microbiol. Rep. 2013, 5, 58-65. [CrossRef] [PubMed]

55. Wirth, T.; Falush, D.; Lan, R.; Colles, F.; Mensa, P.; Wieler, L.H.; Karch, H.; Reeves, P.R.; Maiden, M.C.; Ochman, H.; et al. Sex and virulence in Escherichia coli: An evolutionary perspective. Mol. Microbiol. 2006, 60, 1136-1151. [CrossRef] [PubMed]

56. Weissman, S.J.; Johnson, J.R.; Tchesnokova, V.; Billig, M.; Dykhuizen, D.; Riddell, K.; Rogers, P.; Qin, X.; Butler-Wu, S.; Cookson, B.T.; et al. High-resolution two-locus clonal typing of extraintestinal pathogenic Escherichia coli. Appl. Env. Microbiol. 2012, 78, 1353-1360. [CrossRef] [PubMed]

57. Alikhan, N.F.; Zhou, Z.; Sergeant, M.J.; Achtman, M. A genomic overview of the population structure of Salmonella. PLoS Genet 2018, 14. [CrossRef]

58. Joensen, K.G.; Tetzschner, A.M.M.M.; Iguchi, A.; Aarestrup, F.M.; Scheutz, F. Rapid and easy in silico serotyping of Escherichia coli isolates by use of Whole-Genome Sequencing data. J. Clin. Microbiol. 2015, 53, 2410-2426. [CrossRef]

59. Larsen, M.V.; Cosentino, S.; Rasmussen, S.; Friis, C.; Hasman, H.; Marvig, R.L.; Jelsbak, L.; Sicheritz-Ponten, T.; Ussery, D.W.; Aarestrup, F.M.; et al. Multilocus sequence typing of total-genome-sequenced bacteria. J. Clin. Microbiol. 2012, 50, 1355-1361. [CrossRef]

60. Camacho, C.; Coulouris, G.; Avagyan, V.; Ma, N.; Papadopoulos, J.; Bealer, K.; Madden, T.L. BLAST+: Architecture and applications. BMC Bioinf. 2009, 10, 421. [CrossRef]

61. Carattoli, A.; Zankari, E.; Garcia-Fernandez, A.; Voldby Larsen, M.; Lund, O.; Villa, L.; Moller Aarestrup, F.; Hasman, H. In silico detection and typing of plasmids using PlasmidFinder and plasmid multilocus sequence typing. Antimicrob. Agents Chemother. 2014, 58, 3895-3903. [CrossRef]

62. Zankari, E.; Hasman, H.; Cosentino, S.; Vestergaard, M.; Rasmussen, S.; Lund, O.; Aarestrup, F.M.; Larsen, M.V. Identification of acquired antimicrobial resistance genes. J. Antimicrob. Chemother. 2012, 67, 2640-2644. [CrossRef]

63. Zhou, Z.; Alikhan, N.-F.; Sergeant, M.; Luhmann, N.; Vaz, C.; Francisco, A.; Carriço, J.A.; Achtman, M. GrapeTree: Visualization of core genomic relationships among 100,000 bacterial pathogens. bioRxiv 2017. bioRxiv:216788. [CrossRef]

64. Hamada, M.; Yamada, K.; Sato, K.; Frith, M.C.; Asai, K. CentroidHomfold-LAST: Accurate prediction of RNA secondary structure using automatically collected homologous sequences. Nucleic Acids Res. 2011, 39, W100-W106. [CrossRef] [PubMed]

65. Benson, G. Tandem repeats finder: A program to analyze DNA sequences. Nucleic Acids Res. 1999, 27, 573-580. [CrossRef] [PubMed]

66. Edgar, R.C. PILER-CR: Fast and accurate identification of CRISPR repeats. BMC Bioinf. 2007, 8, 18. [CrossRef] [PubMed]

(C) 2020 by the authors. Licensee MDPI, Basel, Switzerland. This article is an open access article distributed under the terms and conditions of the Creative Commons Attribution (CC BY) license (http://creativecommons.org/licenses/by/4.0/). 